\title{
Distribution of Dialects and Sources of Population in Lianjiang Area, Guangdong, China
}

\author{
Jianya Zhang \\ College of Liberal Arts \\ South China Normal University \\ Guangzhou, China 510006
}

\author{
Weiming Peng \\ College of Liberal Arts \\ South China Normal University \\ Guangzhou, China 510006
}

\begin{abstract}
The Lianjiang area of Guangdong, China has been a land belonging to NanYue since ancient times, where there are various and different dialects, mainly including Cantonese, Min and Hakka. Cantonese is considered the regional dialect. Dialects in this area are staggered and complicated. The population of various dialects is related to the migration of people caused by historical reasons. The time can be traced back to the Song, Yuan, Ming and Qing dynasties.
\end{abstract}

Keywords-dialect distribution; population source; Guangdong of China; Lianjiang city

\section{INTRODUCTION}

Dialects are the product of social history. The division of society can make the language fission and produce dialects. Population migration and cultural dissemination due to historical reasons promote the development of dialects. West Guangdong has been Baiyue area since ancient times, and the people of Han nationality from Northern China moved to Lingnan has been recorded since the Qin Dynasty. [1] After the Tang and Song Dynasties, the population movement in southern China was very active, then it formed the main period of population growth in Lingnan. The city of Lianjiang in the west of Guangdong, where there has a long history and a rich variety of dialects, was the land belonging to Nanyue in the early years of the Western Han Dynasty. This paper uses it as a object to explore the relationship between dialect formation and population sources. Lianjiang City is located in the northern of Leizhou Peninsula, with east to Huazhou City of Maoming, south to Suixi County, bordering Hepu, Luchuan and Bobai counties of Guangxi Zhuang Autonomous Region in the west and north, southeast to Wuchuan and Potou Districts, southwest to the coast of Beibu Gulf. The city's administrative division was recently updated in 2004. At present, there are 3 streets (Luozhou, Chengbei, Chengnan) and 18 towns (Shijiao, Hechun, Liangdong, Shicheng, Xinmin, Anpu, Yingzai, Cheban, Hengshan, Gaoqiao, and Heliao, Ya Tang, Qingping, Shiling, Shijiao, Jishui, Changshan, Tangpeng).

\section{DisTRIBUTION OF LIANJIANG DIALECTS}

Current dialects applied within Lianjiang mainly include Cantonese, Hakka and Min dialect, in addition, there are Hailiao dialect and Diliao dialect, which all belong to Cantonese family. Hailiao dialect is also called Hai dialect in local area. The Hai dialect, with pronunciation similar to the
Cantonese of Liancheng Town, is more preferred to the written language, while the Cantonese is relatively colloquial, but this does not affect the communication between Cantonese speakers and those who speak Hai dialect.

Cantonese is mainly distributed in the six towns of Anpu, Yingzai, Shicheng, Xinmin, Liangdong, and Jishui in the south of Lianjiang. In addition, Qingping, Hengshan, Yatang, Gaoqiao and Shiling towns also have small quantity distributions. Cantonese is the main dialect most widely distributed in Lianjiang, with a population of more than 600 thousand. The "Shicheng County Annals" of the Republic of China stated that the Shicheng (now Lianjiang) Cantonese was known as a "guest language" because many immigrants from the central and southern Guangdong of the Ming and Qing dynasties moved into Shicheng continuously. After that, the Cantonese spoken by immigrants merged with local Cantonese each other and made change to form the Cantonese language of the towns of Lianjiang County today. Most of the immigrants engage in commerce and trade activities, and their commercial port are concentrated in the towns of the northern part of the Leizhou Peninsula. With the increase of economic strength, the power and influence of immigrants have surpassed those of residents living in fishing and farming in the early years, naturally, their spoken Cantonese has gradually become a local dialect. Lianjiang Cantonese today is generally represented by Shicheng dialect. In addition, both the Hai dialect and the Diliao dialect, mainly used in Lianjiang coastal area with a population of about 40 thousand, belong to the Cantonese family, mainly including the villages of Pingdong, Deyao, Dachong, Jinping, Podu and Hongzhai of Gaoqiao Town, Paili, Qingtang of Hengshan Town, and Xiayang of Yingzai Town.

Hakka dialect, applied by about 0.6 million people, is mainly distributed in five towns in the west and north, including Tangpeng, Shijing, Heliao, Changshan and Shijiao, which are pure areas of Hakka dialect. In addition, most of the villages in Hechun, Shiling, Qingping, Gaoqiao, Yatang, and Jishui, as well as a small number of rural villages in Cheban, Yingzi, and Anpu also have Hakka people.

Min dialect, applied by about 0.3 million people and commonly known as "Lei dialect" or "Li dialect" by local people in Lianjiang, is mainly distributed in the four towns of Hengshan, Xinmin, Liangdong and Anpu. In addition, there were small quantity distribution in the villages of Shiling, Yingzai, Yatang, Gaoqiao and Heliao. In the early years, the 
ancestors of Min speakers through the sea lane entered the Leizhou Peninsula and later to Lianjiang. After immigrating to naturalization, they chose to live in the coastal areas, with agriculture and fishery for a living. Due to the low mobility of the population, the original Min dialect spoken by immigrants were retained and evolved into the local language after a long period of time. Comparing Li dialect in Lianjiang and Lei dialect in Leizhou City, it is generally believed that the latter is more "soft" in pronunciation.

\section{Formation OF LiAnJiAng Dialects AND THE SOURCE OF POPULATION}

According to the "Shicheng County Annals", volume two, "A Survey of Geography" revised by Zhong Xizhuo recorded: "There are three languages in the prefecture, one is a Guest language(Cantonese), applied within a large proportion of the city center and the south, and a small part of the West. This language is similar to the one in guangzhou. The Second is $\mathrm{Ai}$ dialect, used in most parts of the East, North and West, with a small part of the city center. They are similar to the Hakka dialect of Jiaying Prefecture. The third type is Li dialect, used in a small part of the South and West, which is similar to the Leizhou dialect." [2] The Lianjiang Cantonese was formerly known as "guests language" before the Republic of China, but in reality it was close to the Cantonese in Guangzhou. "Ai dialect" is a kind of Hakka dialect and is divided into two categories in Lianjiang: one is those less affected by other dialects and the other is those more affected by other dialects with more dispersed population. The Li dialect, belonging to the Min dialect family, is closely related to the Leizhou dialect. The old county annals last mentioned Li dialect, indicating that the number of Li dialect speakers used in the local area is small and the range of using region is relatively small.

\section{A. The Formation of Cantonese}

In the early days, Cantonese-speaking people came to Lianjiang city to do business while Fujian immigrants living in Leizhou Peninsula engaged in agriculture and fishery. With their arrival, Canton dialect has gradually become popular, and formed the earlier cities such as Meilu and Wuchuan, etc. During the Yuan, Ming, and Qing Dynasties, the population of the Cantonese-speaking areas of Shunde, Enping, and Gaozhou also moved to the countryside of Lianjiang County and Wuchuan in succession, so that the number of Cantonese speakers continued to increase. Although these immigrants arrived late, most of them engaged in commercial trade activities, and mainly concentrated in the town center of leizhou peninsula, with the forces and impacts were greater than those of the fujian immigrants that had earlier moved for farming and fishing. Nowadays, the Shicheng and Anpu Town have become the commercial and trade centers of the county with tens of thousands of residents, while the Canton dialect used mainly in the town was gradually formed during the early migration process. In the 18th year of the Qing dynasty, "Shicheng County Annals", written by Jiang Tinggui and Chen Lanbin, Volume Nine "Ji Shu Zhi" recorded: "During the Age of Emperor Tongzhi(同治) in the Qing Dynasty, from September to November 1866, Guangdong governor Jiang
Yifeng sent more than 4,000 immigrants in the Enping and Kaiping areas to Leizhou, and also to the Gaozhou area. By 1869, immigrants were once again allocated to Shicheng County in western Guangdong." [3] Later the "Shicheng County Annals" revised by Zhong Xizhuo in the Republic of China, volume two recorded that Shunde accent was still used by people relocating from Shunde to Shicheng, while the Dongguan accent also remained in those Dongguan immigrations.

\section{B. The Formation of Min Dialect}

Hokkien immigrants entered the Lianjiang area of the west Guangdong earlier usually through sea route and lived in the coastal areas, engaged in agricultural and fishery. "The History of Chinese Migrants" volume four states: "The Leizhou district in the modern Min dialect family include the current cities of Zhanjiang, Suixi, Lianjiang, Haikang, Xuwen, Zhongshan, Yangjiang, Dianbai and Wuchuan, and most of them already have a set number of Hokkien immigrants in the Song Dynasty." [4]

In addition, the literati relegated to the west of Guangdong also recorded the source of the local population. For example, the book "LING WAI DAI DA" by Zhou Qufei in the Song Dynasty divided the population of Nanyue, where many ethnic groups were living together, into five types: The first is natives, namely indigenous people of Nanyue areas. The second is the northern Chinese immigrants who came because of the war. The third is the people of $\mathrm{Li}($ 黎) nationality. Scholars have different opinions about the ethnic origins of the $\mathrm{Li}$ people currently. "Guangdong xinyu", written by Qu Dajun at the turn of Ming and Qing Dynasties, explained the relationship between " $\operatorname{Li}\left(\right.$ 黎 $^{\prime}$ " and " $\mathrm{Li}($ 俚 $)$ " and pointed out that the slang of Nanyue areas was the $\mathrm{Li}$ nationality as the Han nationality called it. Later scholar Yang Chengzhi also agreed with Qu Dajun. He believes that the $\mathrm{Li}($ 俚 $)$ people were the $\mathrm{Li}($ 黎) nationality migrated to Hainan Island. But the Chinese historian Luo Xianglin believed that the "Li people" were "Yue people", and both of them belonged to the same family. The fourth type is the immigrants who were ordered to move for evacuate the population. It was passive migration. Qu Dajun thought that they were Hokkien people. In fact, people in Nanyue areas who mainly depended on cultivated land to made a living were mostly Hakka. So we consider that the fourth kind of people should be mostly Hakka and just a few Hokkien. The fifth kind is the people of $\operatorname{Dan}($ 疍), namely the boat dwellers, is a Chinese clan lived in the coastal areas with a unique history and culture.

Moreover, the genealogy of the local people also recorded the origin of the early migrants in Lianjiang, for example, "Huang Clan Genealogy of Shangxian town in Lianjiang", the Volume 1 preface by Shaoxiong recorded: The ancestor of Shangxian County was Huang $\mathrm{Yu}$, who came from south Fujian of the Yuan dynasty and was appointed as the county magistrate of Shicheng after becoming a Ju-ren (the victor of provincial examination in imperial examination). 
TABLE I. MigRation ReCords in SOME Genealogies of the Min Dialect Families in Lianjiang

\begin{tabular}{|l|l|l|l|l|}
\hline $\begin{array}{c}\text { Last } \\
\text { Name }\end{array}$ & Migration Time & \multicolumn{1}{c|}{$\begin{array}{c}\text { Settlements } \\
\text { (County/Town) }\end{array}$} & \multicolumn{1}{c|}{ Origin } & \multicolumn{1}{c|}{$\begin{array}{c}\text { Ancestor } \\
\text { (Name) }\end{array}$} \\
\hline Luo & Song Dynasty & Hengling & Putian Fujian & Luo Tingyu \\
\hline Liang & $\begin{array}{l}\text { Southern Song } \\
\text { Dynasty }\end{array}$ & Shicheng & Putian Fujian & Liang Zhongzhen \\
\hline Huang & Yuan Dynasty & Shangxian & Putian Fujian & Huang Yu \\
\hline Chen & Yuan Dynasty & Shicheng & Fuzhou Fujian & Chen Zhongzhi \\
\hline Lin & Ming Dynasty & Shiling & $\begin{array}{l}\text { Chaozhou } \\
\text { Guangdong }\end{array}$ & Lin Gong \\
\hline Liao & Ming Dynasty & Xinmin & Fuzhou Fujian & Liao Shanrong \\
\hline
\end{tabular}

\section{The Formation of Hakka Dialect}

Hakka is an ethnic group that moves from north to south in China, most of the dwellings are confused with other ethnic groups. There are many historical records currently available of Hakka ancestors entering the western part of Guangdong. The records of the "County Established Chronicle" of "Chixi County Chronicle, Volume 8" written by Lai Jixi and revised by Wang Dalu are related to the migration of Hakka to Leizhou in the past: "In the Early Qing Dynasty they relocated to Panyu, Dongguan, Xiangshan, Zengcheng, Xin'an, Huaxian, Qingyuan, Longmen, Conghua, Sanshui, Xinning, which belongs to Guangzhou prefecture. At the same time, somebody went to Gaoyao, Guangning, Xinxing, Sihui, Heshan, Gaoming, Kaiping, Enping, and Yangchun, which are under the jurisdiction of Zhaoqing Prefecture, the farthest reached
Gaozhou and Leizhou. " [5] Later, the historian Luo Xianglin's research on the migration of Hakka held that the fifth migration was the important stage when the Hakka entered the western Gao, the Lei, and the Qin prefecture because of the growing population of Hakka lead to the competition with indigenous peoples. By 1867, the governor of Guangdong Province, Jiang Yili, assigned the Hakka immigrations to make a living in other parts of western Guangdong. This incident was known as the "Guangdong West Road Incident". [6] After that, the Hakka immigrants moved southwestwards to reach Xinyi of Gaozhou and Xuwen of Leizhou as the majority, and the farthest even reached Ya County and Ding'an County of Hainan Island. Scholar Zhang Zhenxing also agreed that the Hakka people in Lianjiang migrated from eastern Guangdong. [7] We collected some of the genealogy of local Hakka families in Lianjiang and compiled them into the "Table II":

TABLE II. Migration ReCORDS IN SOME GENEALOGIES OF THE HAKKa DialeCt FAMILIES IN LianJiang

\begin{tabular}{|l|l|l|l|l|}
\hline $\begin{array}{c}\text { Last } \\
\text { Name }\end{array}$ & Migration Time & \multicolumn{1}{|c|}{$\begin{array}{c}\text { Settlements } \\
\text { (County/Town) }\end{array}$} & \multicolumn{1}{c|}{ Origin } & \multicolumn{1}{c|}{$\begin{array}{c}\text { Ancestor } \\
\text { (Name) }\end{array}$} \\
\hline Liufu & Ming Dynasty & Shijiao & $\begin{array}{l}\text { Tingzhou } \\
\text { Fujian }\end{array}$ & Liufu Luan \\
\hline Liao & Ming Dynasty & Hechun & $\begin{array}{l}\text { Tingzhou } \\
\text { Fujian }\end{array}$ & Liao Yuchen \\
\hline Tang & Ming Dynasty & Tangpeng & $\begin{array}{l}\text { Tingzhou } \\
\text { Fujian }\end{array}$ & Tuang Yuanda \\
\hline Wen & Ming Dynasty & Changshan & Ninghua Fujian & Wen Jingquan \\
\hline
\end{tabular}

Based on the genealogies we have collected, the ancestors of Lianjiang who spoke Hakka dialects were mostly immigrants who migrated from the Hakka base camp of Tingzhou, Shanghang and Ninghua in Fujian province, and some have been transferred from Meizhou, the eastern Guangdong province. Therefore, the Lianjiang Hakka can basically communicate with Hakka in Meizhou today.

\section{CONCLUSION}

China is a multi-dialect country, and Chinese dialects have gradually evolved with the migration of people and the spread of culture. In general, the relationship between regional dialects and immigrants is roughly represented by: First, people speaking A dialects migrated to different regions due to disasters, war, etc., and coexisted with the original indigenous language to form a dialect region. Second, some of the residents of Part A dialect migrated to other places for historical reasons. Over time, a third mixed dialect was produced. The pattern of dialects in the Lianjiang region today is mainly manifested in the above first case. The three main dialects of Lianjiang mentioned above interact with each other, among which Cantonese is the most influential. The locals generally regard Cantonese as a dominant dialect, that is, the speakers who speak Hakka and Min dialects can basically speak Cantonese, but in turn, people who speak Cantonese may not speak the Hakka or the Min dialect. Based on the use of population in all aspects of Lianjiang, the population of Cantonese and Hakka is evenly matched, while the people of Min is the least. However, the population quantity sometimes does not actually determine the influence of dialects. 


\section{REFERENCES}

[1] Wang Zijin, "Lingnan Immigration and the Expansion of Han Culture A Comprehensive Survey of Archeological Data and Literature," Journal of Sun Yat-Sen University(Social Science Edition), Vol.50, No. 4, 2010, pp. 110-116.

[2] Jiang Xun, Zhong Xizhuo, Shicheng County Annals, Chengwen Publishers, 1931, p. 153.

[3] Jiang Tinggui, Shicheng County Annals, 18 years of the Age of Emperor Guangxu(光绪), 1892, p. 61.

[4] Ge Jianxiong, Wu Songdi, Cao Shuji, The History of Chinese Migrants, Fujian People's Publishing House, 1997, p. 185.

[5] Lai Jixi, Wang Dalu, Chixi County Chronicle, Republic of China (1920) Revised, fifteen (1926) years of photocopying, p. 61.

[6] Luo Xianglin, An Introduction to Hakka Research, Shanghai Literature and Art Publishing House, 1992 (photocopy), p. 62.

[7] Zhang Zhenxing, "Distribution of Dialects in Leizhou Peninsula, Guangdong Province", Dialect, No. 3, 1986, pp. 204-218. 